\title{
Embolic Stroke and Pulseless Right Arm in a Schoolgirl with Arterial Thoracic Outlet Syndrome: A Case Report
}

\author{
AMINUR RAHMAN ${ }^{1}$, FIROZ AHMED QURAISHI ${ }^{2}$, UTTAM KUMAR SAHA $^{3}$, MALIHAHAKIM $^{4}$, \\ AFZAL MOMIN ${ }^{5}$, MD.NURULAMIN MIAH $^{6}$
}

\begin{abstract}
:
A rare clinical presentation arterial Thoracic outlet syndrome (TOS) is described in a young school-girl. TOS causing distal; disease is a rare cause of artery to- artery embolic stroke. Brain-stem ischemic stroke is a result of compromise to the posterior circulation. This is often due to antegrade embolism from the heart or proximal vessels. Retrograde blood flow has been described in the subclavian artery, thus making the distal subclavian artery a source of possible retrograde embolism to carotid circulation ${ }^{1}$. Clinical presentation also included left hemiparesis caused by right subclavian artery thrombosis and retrograde embolizatoin of thrombus via common carotid artery to the right middle cerebral artery (MCA) distribution².
\end{abstract}

Keywords: Thoracic outlet syndrome; arterial Thoracic outlet syndrome; subclavian artery

\section{Case Report:}

A15 years old girl normotensive nondiabetic right handed school-girl admitted with the history of episodic unilateral headache followed by sudden onset of left side weakness with focal convulsion. She had a episodic headache 6 months back. Each episode started with blurring of vision followed by unilateral throbbing headache associated with vomiting persisted for several hours \& relieved spontaneously. Headache was aggravated by light and sound and patient felt comfortable in dark place. One month back patient developed sudden severe global headache without vomiting and suddenly fell in the ground followed by left sided weakness and repeated convulsion on the left side of body. She had no history of eye or neck pain nor any history of fever or joints pain, morning stiffness, rash, oral ulcer, hair loss, photosensitivity, abdominal pain, leg ulcer, bone pain, chest pain, palpitation, exertional dyspnoea, migratory arthritis, or any bleeding manifestations. She did not give any history of right arm pain suggestive of claudication or transient loss of consciousness and did not have any permanent neurologic deficit previously and she had not sought any medical advice. She did not have any history of relevant medication \& substance abuse. All family members are healthy and alive except one of her paternal uncle had stroke 3 months back at the age of 55 . Physical examination reveals good nutrition with normal peripheral pulses but absent right radial \& brachial pulse. Her blood pressure is normal with no carotid, supraclavicular or renal bruit. Her neurological examination reveals left sided upper motor neuron type facial palsy. Tone is increased on the left side with diminished muscle power (MRC Grade o). All deep tendon reflexes are exaggerated on left side with left plantar extensor. Gait is hemiplegic.

Investigations regarding- CT scan of brain done immediately after admission into hospital shows Infarction of right temporoparietal region that is right MCA territory and bilateral cervical rib on chest Xray. Subsequent MRI and MRA revealed infarction of right MCA with normal posterior circulation.

1. Registrar, Department of Neurology, Sir Salimullah Medical College and Mitford Hospital, Dhaka

2. Professor\& Head, Department of Neurology, Sir Salimullah Medical College, Dhaka.

3. Assistant Professor, Department of Medicine, Sir Salimullah Medical College, Dhaka

4. Professor, Department of Neurology, Shaheed Suhrawardy Medical College, Dhaka.

5. Assistant Professor, Department of Neurology, Shaheed Suhrawardy Medical College, Dhaka

6. Assistant Professor, Department of Neurology, Sir Salimullah Medical College, Dhaka. 
Duplex study neck vessels is normal .Duplex study territory of upper right arm vessels- shows Short right brachial artery with suspected early bifurcation at the level of mid-arm. Non-detectable right radial artery .Digital Subtraction Angiography (DSA) is done after 2 months shows normal cerebral angiogram with normal right common carotid artery and its bifurcation and left common carotid and left subclavian artery angiography are normal . There is an obstruction of right subclavian artery with superadded thrombus just after where clavicle and first rib crosses each other. Multiple tandem obstruction is seen shortly thereafter and right axillary artery is very narrow calibered and totally occluded just above elbow. It is reformed from

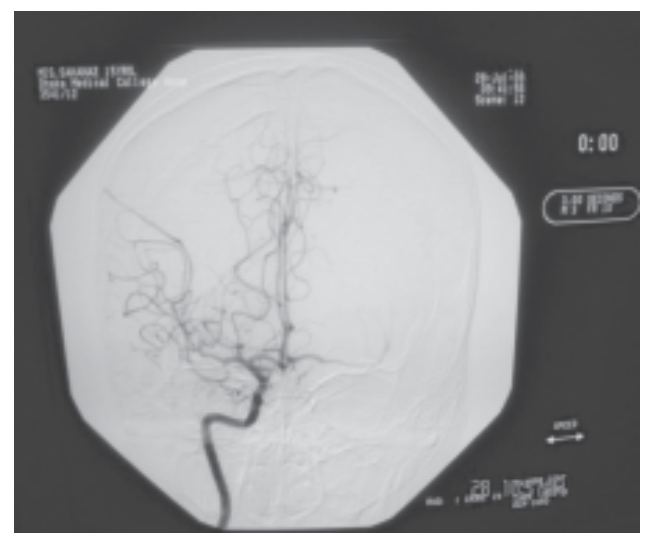

Fig.-1: RICA angiogram, AP view shows Normal Intracranial circulation

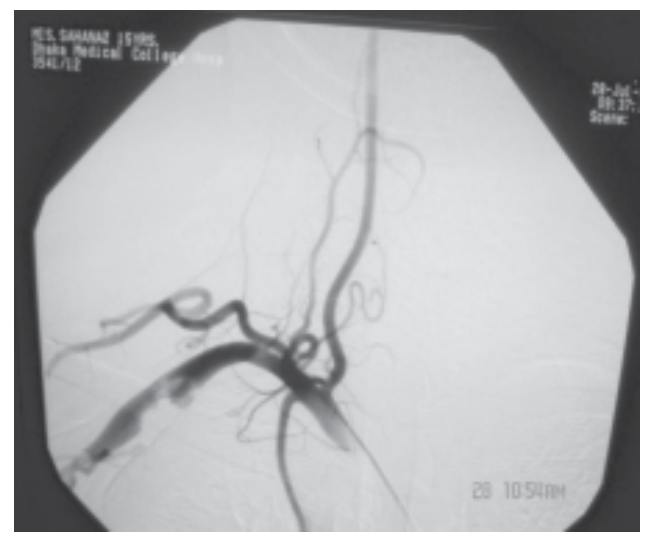

Fig.-2: Right subclavian artery angiogram shows normal vertebral ostium. There is an obstruction with superadded thrombus just after where clavicle and first rib crosses each other. There is multiple tandem obstruction shortly there after

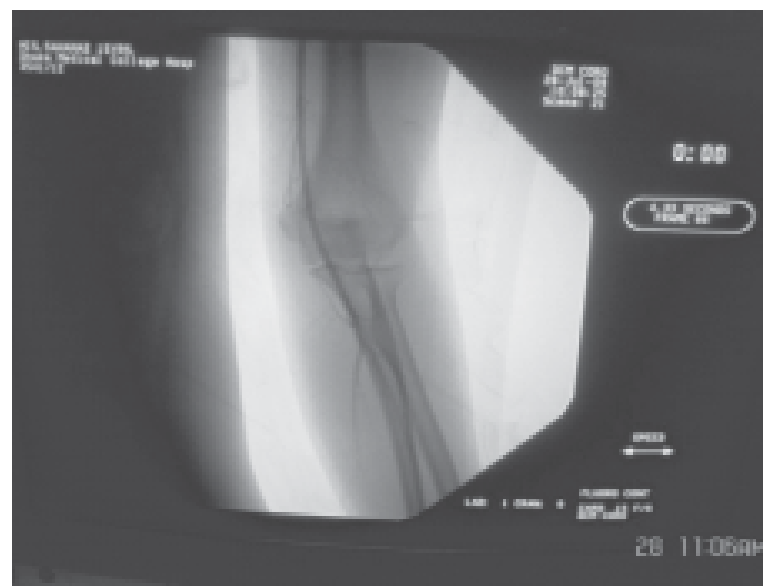

Fig.-3: Right axillary artery angiogram shows very narrow caliber and finally total occlusion just above elbow. It is reformed from collateral below the elbow and divides into normal looking radial and ulnar branches where distal flow is poor.

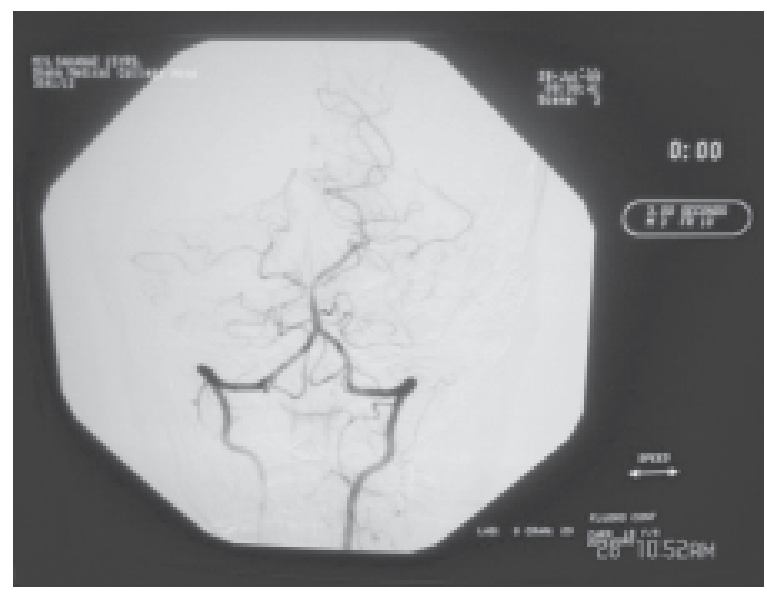

Fig.-4: Vertebral artery angiogram. AP view shows normal intracranial circulation

collaterals below the elbow and divides into normal looking radial and ulnar branches where distal flow is poor. Transthoracic echocardiography, thrombophilia screen, and C-reactive protein were normal. A screen for arrhythmias was negative. However the test for anti ds DNA, anticardolipin antibody. antiphospholipid antibody, protein C, protein $\mathrm{S}$ all are negative. p-ANCA, c-ANCA and $S$ homocysteine are normal. 


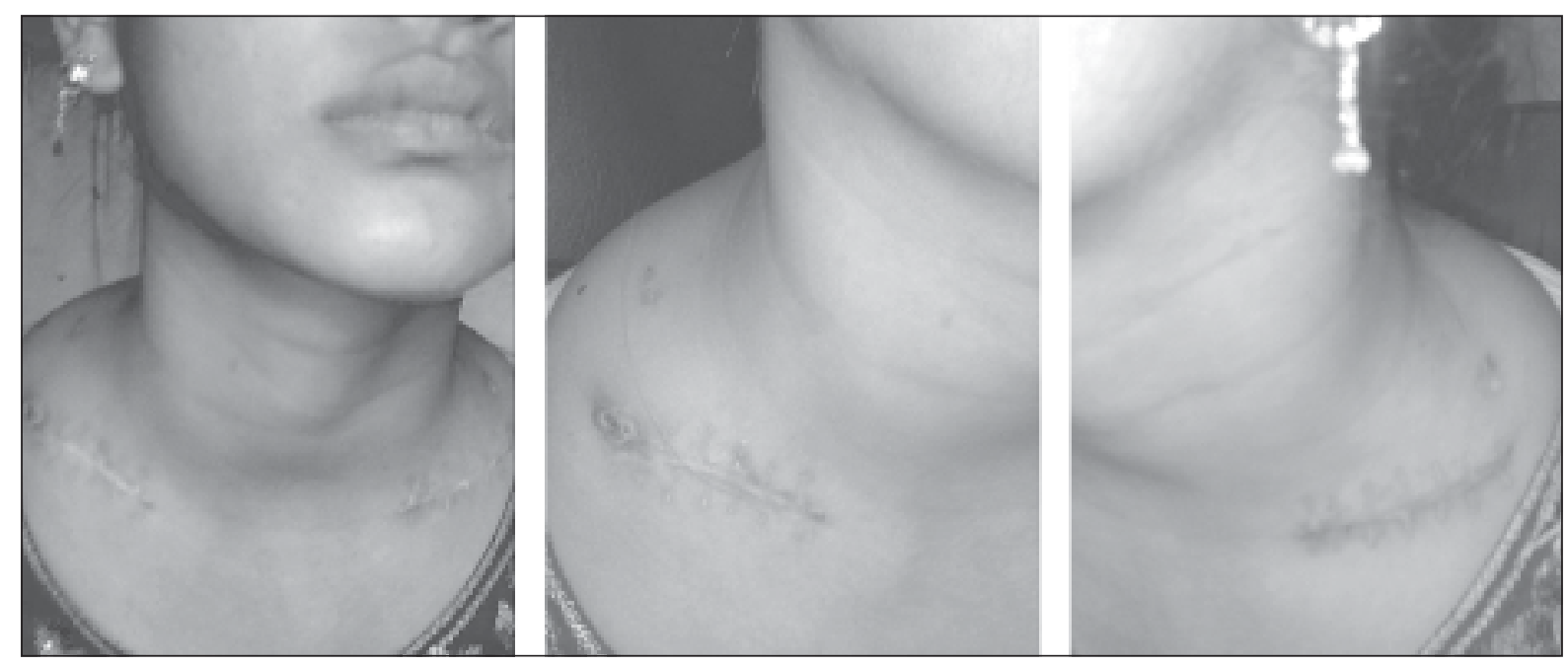

Fig 5: surgical intervention Of TOS in our patient - supraclavicular approach.

\section{Discussion:}

The abrupt onset of left hemiparesis with focal convulsion in this patient suggested ischemic stroke involving right temporoparietal region which was confirmed by CT scan ${ }^{2}$. The commonest cause of ischemic stroke in a young patient is embolism, originating from the heart ${ }^{3}$. However, clinical evaluation, normal electrocardiographic assessment, and a negative transthoracic echocardiogram made the heart an unlikely source of embolism in this patient. Less commonly, arteryto-artery embolism, especially from the proximal circulation and often secondary to dissection, is an important cause of stroke in the young ${ }^{3}$. Free flow of contrast in the proximal vessels, including the cervical vertebral artery and innominate artery, made embolism from the proximal vessels unlikely. X-ray cervical spine suggested a right cervical rib causing thoracic outlet syndrome (TOS) and distal subclavian artery disease. There are 2 possible mechanisms of cerebral embolism in this case: retrograde propagation of a thrombus and retrograde cerebral embolism. The subclavian artery may be subject to trauma by a cervical rib, resulting in progressive stenosis and occlusion. Typically, there may be poststenotic dilation and aneurysm formation. A mural thrombus may form at the site of the compression or in the aneurysmal segment. A thrombus thus formed has an opportunity of extending proximally and embolizing via the right common carotid artery to occlude right middle cerebral artery (in this patient). Retrograde propagation of a thrombus has been described ${ }^{4}$.
Theoretically subclavian artery flow patterns measured using doppler ultrasound indicated both antegrade and retrograde flow occurring across both subclavian lumina in cardiac systole with small amounts of retrograde flow in diastole. Retrograde flow in diastole was greater in the occluded subclavian artery compared to the left. It was noted that retrograde flow increased on extreme rotation of the neck. Retrograde flow could carry an embolus toward the origin of the vertebral artery, and the next cardiac contraction would propel the embolus into the vertebral circulation. Previous case reports have demonstrated bidirectional flow in normal as well as occluded subclavian arteries ${ }^{5}$. These too have demonstrated greater retrograde flow in the occluded vessels suggesting greater reverse flow due to increased peripheral resistance. TOS refers to compression of the neurovascular structures in the area just above the first rib and behind the clavicle. The subclavian artery is affected in $1 \%$ of cases of TOS. Cervical ribs and the first anomalous rib are rare conditions, present in approximately $1 \%$ of the population and in $4.5 \%$ of patients with TOS $^{6}$.

The type of cervical rib is of significance in arterial complications ${ }^{7}$. It has been established that short and incomplete ribs preferentially produce neurologic complications by nerve compression, while long or complete ribs as seen in this patient have arterial complications. Thromboembolism to the forearm and digits is the most typical clinical presentation of patients with distal subclavian artery 
disease due to TOS. Due to collateral formation, ischemic symptoms may be mild or absent. The presence of collaterals also suggests longstanding thrombosis of the distal subclavian artery.

Previous case reports have described distal subclavian artery disease secondary to TOS causing cerebral embolism ${ }^{8}$. The majority of these cases described right TOS leading to cerebral embolism, involving commonly the right middle cerebral artery. This phenomenon is possibly due to the anatomic characteristics of the right carotid artery branching from the brachiocephalic artery and the differences in caliber of the common carotid and vertebral arteries with reduced resistance seen in the larger common carotid artery.

Surgical intervention is indicated in vascular TOS as it is typically resistant to conservative management. The surgical treatment of arterial TOS includes the excision of the cervical and/or first rib, followed by reconstructive vascular procedure, such as resection and anastomosis or replacement with vein or prosthetic grafts ${ }^{9}$. Two common approaches to TOS are the supraclavicular and transaxillary approaches. The advantage of the supraclavicular approach is that it allows good exposure of the brachial plexus, medial two thirds of the first rib, and the cervical rib, if present. As a result, first rib resection or anterior and middle scalenectomies can be done with this approach. In our patient surgeons preferred the supraclavicular approach.

The success rates of the supraclavicular and transaxillary approaches defined as complete to partial resolution of symptoms are $87.5 \%$ to $89 \%$ and $81 \%$ to $93 \%$, respectively ${ }^{10}$. Potential complications from TOS decompression surgeries include major neurovascular injuries, Horner's syndrome and phrenic nerve, long thoracic nerve, supraclavicular nerve, and intercostal brachial nerve injuries ${ }^{11}$.

Surgical treatment may be considered in a patient with occlusive distal subclavian artery disease secondary to a cervical rib in order to prevent recurrent embolism ${ }^{12}$.

\section{References:}

1. Gooneratne I K, Gamage R, Gunarathne K.S, Pearls \& Oy-sters: Distal subclavian artery A source of cerebral embolism Neurology 73, 2009; e11-12.
2. Kasper DL, Fauci AS, Longo DL, Braunwald E, Hauser SL, Jameson JL. Cerebrovascular diseases. In: Harrison's Principles of Internal Medicine, 17th ed. New York: McGraw-Hill; 2009:2372-93.

3. Hart RG, Freeman GL. Stroke in young people: the heart of the matter. West J Med 1987; 146:596-7.

4. Caplan LR. Posterior Circulation Disease: Clinical Findings, Diagnosis, and Management. Cambridge, MA: Blackwell Science; 1996.

5. Prior AL, Wilson LA, Gosling RG, Yates AK, Ross-Russell RW. Retrograde cerebral embolism. Lancet 1979;851: 1044-7.

6. Sanders RJ, Hammond SL. Management of cervical ribs and anomalous first ribs causing neurogenic thoracic outlet syndrome. J Vasc Surg 2002;36:51-6.

7. Gruber W. Ueber die Halsrippen des Menshen mit verglerchendanatomischen Bermerkungen. St Petersburg: Memoires de l'Academie Imperial Scientia; 1869;2:7-27.

8. Yamaguchi $\mathrm{R}$, Kohga $\mathrm{H}$, Kurosaki M. Acute basilar artery occlusion in a patient with subclavian artery occlusion due to first rib anomaly. Neurol Med Chir (Tokyo) 2008;48:355-8.

9. Davidovic LB, Kostic DM, Jakovljevic NS. Vascular thoracic outlet syndrome. World J Surg. 2003;27:545-50.

10. Lee TS and Hines GL. Cerebral Embolic Stroke and Arm Ischemia in a Teenager With Arterial Thoracic Outlet Syndrome: Vascular and Endovascular Surgery 2007; 41(3):254-7

11. Maxey TS, Reece TB, Ellman PI. Safety and efficacy of the supraclavicular approach to thoracic outlet decompression. Ann Thorac Surg. 2004;40:599-603.

12. Bertoletti G, Varroni A, Capoccia L. Surgical treatment of thoracic outlet syndrome: immediate and midterm results. Arch Med Sci 2007;3:355-9. 